\title{
Statin Pharmacology Revisited
}

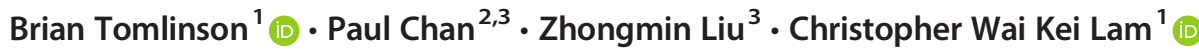

Published online: 4 March 2020

(C) Springer Science+Business Media, LLC, part of Springer Nature 2020

We read with interest the review on statins by Azemawah and colleagues, but we considered that there were certain important errors and omissions [1]. The authors provided several conflicting statements about the association of new onset diabetes mellitus (NODM) with statin treatment, and they concluded in the abstract that this is rare, controversial, and not proven. We consider this is well proven from the large clinical trials with statins, observational studies, and the Mendelian randomization study [2]. Notably, the Mendelian randomization study also predicted that the loss of function variants in the gene for proprotein convertase subtilisin-kexin type 9 (PCSK9) were also associated with NODM [3], but this has not been seen in the clinical trials with the monoclonal antibodies inhibiting PCSK9. These antibodies only inhibit circulating PCSK9, and it will be interesting to see if inclisiran, which inhibits intracellular PCSK9 production, is associated with NODM in the long-term studies.

The risk of NODM with statins increases in subjects with the metabolic syndrome or prediabetes and with increasing intensity of statin therapy reaching $34 \%$ increased risk with

Brian Tomlinson and Paul Chan contributed equally to this work.

Christopher Wai Kei Lam

wklam@must.edu.mo

Brian Tomlinson

btomlinson@must.edu.mo

Paul Chan

chanpaul@w.tmu.edu.tw

Zhongmin Liu

liu.zhongmin@tongji.edu.cn

1 Faculty of Medicine, Macau University of Science and Technology, Avenida Wai Long, Taipa, Macau 999078, China

2 Department of Internal Medicine, Division of Cardiology, Wan Fang Hospital, Taipei Medical University, No. 111, Sec.3, Hsin-Lung Road, Wen-Shan District, Taipei 116, Taiwan

3 Research Center for Translational Medicine, Shanghai East Hospital Affiliated to Tongji University School of Medicine, No 150, Jimo Road, Shanghai 200120, China atorvastatin $80 \mathrm{mg}$ [4]. The authors stated that pravastatin has never been shown to have any diabetic effects, but actually there was a significant $32 \%$ increase in NODM with pravastatin $40 \mathrm{mg}$ compared to placebo in the PROSPER (PROspective Study of Pravastatin in the Elderly at Risk) trial [5]. Overall, the least risk of NODM is seen with pitavastatin [4], which was not mentioned in the review, although it was approved by the FDA in 2009. Pitavastatin is also the statin with the least propensity for drug-drug interactions and the greatest in vitro binding affinity to HMG-CoA reductase of all the currently available statins.

The small excess of NODM with statins was detected by large-scale randomized trials, as was a small excess of hemorrhagic stroke (not mentioned in the review), but these trials failed to detect a long-term increase in muscle-related symptoms with statins [6]. The role of statins in the milder forms of muscle symptoms is disputed and has been attributed to a nocebo effect, but there is no dispute that statins can cause severe myopathy and potentially fatal rhabdomyolysis, which appears to be related to the statin concentration in skeletal muscle reaching a critical threshold.

Azemawah and colleagues discuss the role of drug metabolizing enzymes in drug interactions with statins but do not mention the important role of drug transporters in statin disposition and toxicity. The genome-wide association study to identify genetic associations of definite or incipient myopathy with $80 \mathrm{mg}$ simvastatin daily (2.3\% over a mean follow-up of 6.7 years) from the SEARCH (Study of the Effectiveness of Additional Reductions in Cholesterol and Homocysteine) trial identified the noncoding rs4363657 single-nucleotide polymorphism (SNP) in the SLCO1B1 gene encoding the organic anion-transporting polypeptide 1B1 (OATP1B1) liver uptake transporter, which was in nearly complete linkage disequilibrium with the functional rs4149056 (c.521T>C, p. Val174Ala) SNP, known to influence the liver uptake of simvastatin acid [7]. Over $60 \%$ of the myopathy cases in that study could be attributed to the $S L C O 1 B 1 \mathrm{C}$ variant.

The influence of the $S L C O 1 B 1$ rs 4149056 SNP on the risk of myopathy with high-dose simvastatin was also the subject 
of a guideline from the clinical pharmacogenomics implementation consortium (CPIC) [8], which recommended avoiding the dose of simvastatin $40 \mathrm{mg}$ in carriers of the $\mathrm{C}$ variant. Genotyping for the rs 4149056 polymorphism is not performed routinely before initiating statin therapy. The frequency of the rs $4149056 \mathrm{C}$ variant is similar in most ethnic groups, but the systemic exposure of simvastatin is also influenced to a lesser extent by the functional rs 2231142 (c. $421 \mathrm{C}>\mathrm{A}$, p. Gln141Lys) variant in the gene for the ATP-binding cassette G2 (ABCG2) efflux transporter, which is more common in east Asian populations than in Caucasians [9].

We recently described a case of simvastatin-induced myopathy in a Chinese diabetic patient who had been taking simvastatin $40 \mathrm{mg}$ daily for 10 years [10]. He developed rhabdomyolysis with no well-established predisposing factor, and genetic analysis showed that he was a carrier of one copy of the $\mathrm{C}$ allele of rs4149056 and two copies of the $A B C G 2$ rs2231142 variant, both of which increase simvastatin acid systemic exposure. The toxicity may have been precipitated by some mild drug interactions or a gradual decline in renal function over the years; but following the CPIC guideline, the patient should never have been started on simvastatin $40 \mathrm{mg}$. The timing of this event illustrates the fallacy of the notion that if a patient has been taking a high dose of simvastatin for over a year, it will inevitably be safe. The risk of severe myopathy is highest in the first few months of therapy and declines thereafter but does not disappear completely even after 1 year, as shown in the analysis from the SEARCH study [7].

In the absence of pretreatment genotyping, we would recommend considering the patient's ethnicity before prescribing the highest doses of statins. The maximum doses of statins approved in Japan are simvastatin $20 \mathrm{mg}$, atorvastatin $40 \mathrm{mg}$, and rosuvastatin $20 \mathrm{mg}$, and we recommend following these as the maximum doses to use in Chinese patients based on the similar pharmacokinetics in Chinese and Japanese subjects [9]. The effect of ethnicity applies irrespective of where the patient resides and all physicians should be aware of this.

Azemawah and colleagues stated that grapefruit juice enhances the efficacy of some statins, such as simvastatin; but the reference they quoted only speculated on this effect, and to our knowledge, there is no clinical study showing that grapefruit juice or other enzyme inhibitors significantly enhance the efficacy of statins, but they do increase the risk of toxicity.
They also stated that "rosuvastatin also carries a greater risk of potentially fatal statin-induced myopathy" without providing any reference to support that statement. We disagree with that statement and we consider that the risk with appropriate maximum doses of rosuvastatin is much less than with simvastatin $80 \mathrm{mg}$, which is a dose we think should never be initiated.

\section{References}

1. Azemawah V, Movahed MR, Centuori P, Penaflor R, Riel PL, Situ $\mathrm{S}$, et al. State of the art comprehensive review of individual statins, their differences, pharmacology, and clinical implications. Cardiovasc Drugs Ther. 2019;33(5):625-39.

2. Mach F, Ray KK, Wiklund O, Corsini A, Catapano AL, Bruckert E, et al. Adverse effects of statin therapy: perception vs. the evidence focus on glucose homeostasis, cognitive, renal and hepatic function, haemorrhagic stroke and cataract. Eur Heart J. 2018;39(27):252639.

3. Ference BA, Robinson JG, Brook RD, Catapano AL, Chapman MJ, Neff DR, et al. Variation in PCSK9 and HMGCR and risk of cardiovascular disease and diabetes. N Engl J Med. 2016;375(22): 2144-53.

4. Thakker D, Nair S, Pagada A, Jamdade V, Malik A. Statin use and the risk of developing diabetes: a network meta-analysis. Pharmacoepidemiol Drug Saf. 2016;25(10):1131-49.

5. Sattar N, Preiss D, Murray HM, Welsh P, Buckley BM, de Craen AJ, et al. Statins and risk of incident diabetes: a collaborative metaanalysis of randomised statin trials. Lancet. 2010;375(9716):73542.

6. Peto R, Collins R. Trust the blinded randomized evidence that statin therapy rarely causes symptomatic side effects. Circulation. 2018;138(15):1499-501.

7. Link E, Parish S, Armitage J, Bowman L, Heath S, Matsuda F, et al. SLCO1B1 variants and statin-induced myopathy-a genomewide study. N Engl J Med. 2008;359(8):789-99.

8. Wilke RA, Ramsey LB, Johnson SG, Maxwell WD, McLeod HL, Voora D, et al. The clinical pharmacogenomics implementation consortium: CPIC guideline for SLCO1B1 and simvastatininduced myopathy. Clin Pharmacol Ther. 2012;92(1):112-7.

9. Tomlinson B, Chan P, Liu ZM. Statin responses in Chinese patients. J Atheroscler Thromb. 2018;25(2):199-202.

10. Chan JCM, Ng M-H, Wong RSM, Tomlinson B. A case of simvastatin-induced myopathy with SLCO1B1 genetic predisposition and co-ingestion of linagliptin and Stevia rebaudiana. J Clin Pharm Ther. 2019;44(3):381-3.

Publisher's Note Springer Nature remains neutral with regard to jurisdictional claims in published maps and institutional affiliations. 